\title{
Migration of Data from One Cloud Server to another Cloud Server using the TCP-IP Protocol
}

\author{
Dinesh Kumar \\ Research Scholar, \\ Banasthali University, \\ Banasthali, Jaipur (Raj.)
}

\author{
Praveen Dhyani, PhD \\ Executive Director, \\ Jaipur Campus Banasthali \\ University, Banasthali, Jaipur
}

\author{
Anuj Kumar Sharma, PhD \\ Associate Professor \& Dean, \\ Om Institute of Management \& \\ Technology, Hisar. (Haryana.)
}

(Raj.)

\begin{abstract}
Another territory of some hugeness that does not include a particular innovation is information administration. A few organizations had their movement specifically attached to their information administration activity, some had administration extends that weren't connected with the relocation, and others had no information administration set up. The remainder of these were $10 \%$ more averse to get on time and on spending ventures than the individuals who had some experience of information administration regardless of the possibility that that governance was not connected with the relocation extend. Truth be told, they prescribe that on the off chance that you have not already selected information administration then an information relocation venture is a decent place to begin in light of the fact that the work that you have to do-profile the information, scrub it, conceivably chronicle and cover it - are all the very same sorts of things that you would do inside an administration activity, in addition to different capacities, for example, developing a business glossary.
\end{abstract}

\section{Keywords}

SQL, Data Migration, IAAS, PAAS, SAAS, Database etc

\section{INTRODUCTION}

Cloud computing is an advancement that customs the web and central remote servers to keep up info and requests. Cloud computing grants clients and associations to use requests without creation and contact their own particular accounts at any computer with web access. This advancement considers extensively more capable bringing together in order to enlist data stockpiling, get ready and information exchange limit.

A straightforward illustration of Cloud computing is Email Server like Gmail, Yahoo mail, MSN and so on. Everything you need is only a web association and you can begin spreading messages. All the Mail and Server management tasks are on cloud and is totally supervised by the cloud organisation supplier like Amazon, Go-daddy and so forth. The customer gets the chance to apply the product alone and appreciate the advantages.

Cloud computing is separated into three portions: "Network" "Storage" and "Application." Every section fills a substitute need and offers altered things for associations and entities far and wide. In June 2011, a training coordinated by V1 initiate that $91 \%$ of senior IT specialists truly don't understand what Cloud processing is and 66\% of senior asset specialists are perfect by the thought, importance the energetic method for the development. In Sept 2011, an Aberdeen Group study found that restrained organizations accomplished all things considered a $68 \%$ expansion in their IT cost in light of the fact that Cloud computing and just $10 \%$ lessening in server farm force costs.

Cloud computing initiates into focus just once you think what IT generally needs: a methodology to build boundary or contain abilities the fly without placing properties into new foundation, preparing new faculty, or permitting new programming. Cloud computing integrates any association based on pay-per-utilization benefit that, increasingly over the Internet, expands its current volumes. Cloud computing is at an primary stage, with a several group of providers extensive and little transmission a huge number of cloud-based organizations, from out and out submissions to capacity organizations to spam sifting. Yes, utility-style base providers are a blend's piece, yet so are Software as a Service suppliers, for example, Amazon.com. Today, mostly, IT must associate to cloud-based managements, however Cloud computing integrators and aggregators are from nowadays developing.

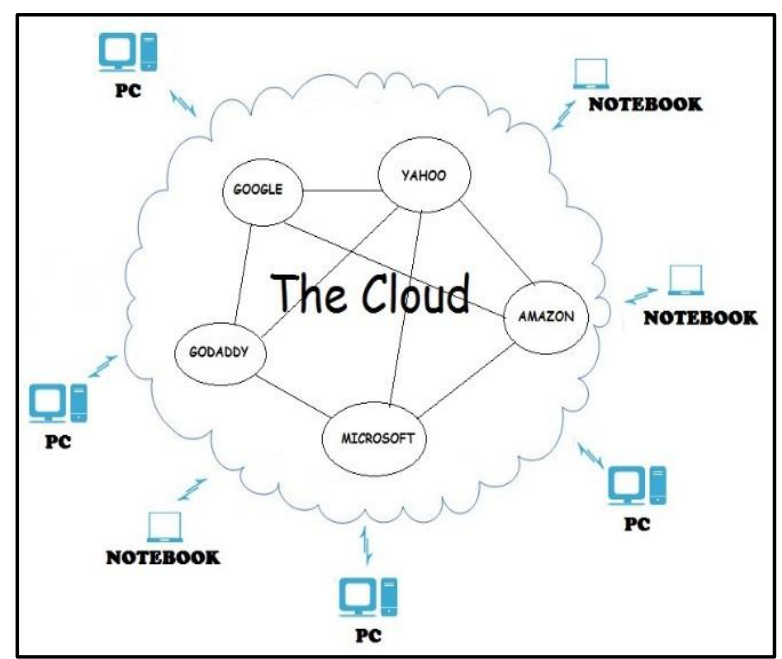

Figure 1 Cloud Computing Environment

Additionally referred to as a system cloud. In information transfers, a cloud refers to an open space on broadcast lines, (for example, B1 or B3) that occurs between the end resolutions of a transmission. Info that is transmitted over a Wide Area Network arrives the organization from one end point developing an ordinary resolution suite, for example, Frame Relay and after that arrives the cloud system where it informs space to other information programs. The information rises up out of the cloud - where it might be typified, interpreted, transported in heap ways - in the similar organization as when it arrived the cloud. A system exists on the grounds that when info is transferred over a portion based system in a package, no two packages will fundamentally take 
after the same physical way. The eccentric territory that the information enters before it is gotten is the cloud.

\section{HISTORY OF RESEARCH WORK}

Another range of some importance that does not include a particular innovation is information administration. A few organizations had their relocation straightforwardly fixed to their information administration activity, some had administration extends that weren't connected with the movement, and others had no information administration set up. The remainder of these were $10 \%$ less inclined to get on time and on spending plan ventures than the individuals who had some experience of information administration regardless of the fact that that governance was not connected with the relocation venture. Actually, they suggest that in the event that you have not already decided on information administration then an information relocation undertaking is a decent place to begin on the grounds that you have to do outline the information, wash down it, potentially file and cover it, in addition to different capacities, for example, building up a business glossary.

Table 1 Stages and their criteria involved in planning the review

\begin{tabular}{|c|l|}
\hline Stages & \multicolumn{1}{c|}{ Criteria } \\
\hline $\begin{array}{c}\text { Identify the need } \\
\text { of Research }\end{array}$ & $\begin{array}{l}\text { - Research gap } \\
\text { - Fair research }\end{array}$ \\
\hline $\begin{array}{c}\text { Specify Research } \\
\text { Question }\end{array}$ & $\begin{array}{l}\text { - Security issues } \\
\text { - Migration to clouds } \\
\text { migration }\end{array}$ \\
\hline $\begin{array}{c}\text { Develop Review } \\
\text { protocol }\end{array}$ & $\begin{array}{l}\text { - Research questions } \\
\text { - Choosing appropriate keyword } \\
\text { - Developing the search strings }\end{array}$ \\
\hline
\end{tabular}

As we have already explained in the abstract that the cloud computing services has been one of the most booming fields of the software development and engineering field. There are several service providers in this field and all of them have certain benefits which are called advantages and certain disadvantages which are called drawbacks. Since the time of the launch of different cloud computing services, critics also came into action. Critics are someone who always tells you that what is better and what is not. Critics are present in each and every field whether you are talking about the entertainment or data services or anything else. Hence as the Go Daddy became the first to launch the cloud computing services, their critics also came into action. In this chapter literature survey will be discussed.

\section{DATA MIGRATION}

With admire to transformation of DB in detailed, Microsoft focus on new technology systems and whole database migrated to Cloud and rest layers access ordinary.
1. Applications of web.

2. Purposes utilized specific departments and by other agencies that are smaller in size, inside organization

Data hubs, essential data placed in the references or user can access the data easily, e.g., on the personal computers on users, and almost always directly access to the DB in the Cloud. Certainly for primary two eventualities, performance issues and complexity as a result of expertise latency challenges after migration of database

In this fast moving world of digitization, cloud computing is becoming an essential part of the daily life, especially when you are related to the business. Keeping the data secure on the server has become one of the major issues. To overcome this problem Cloud Computing has emerged into the world but it is quite expensive. As if for now, one protocol architecture does not allow another protocol architecture to communicate directly, so there is a need to find a unique crossbreed application architecture which can convert one protocol architecture into an intermediate architecture which is completely understandable by another service architecture so that anyone can move up the entire data architecture along with the data from one server end to another without any data loss.

\subsection{Objective}

- To exchange current server designing protocol into reference of middle service.

- To modify the service provider reference to different platform of cloud to make specific exclusion of architecture/structure from single platform to cloud.

- To generate service reference in line with Go Daddy protocol architecture for information elimination from home server to cloud.

- To design and develop godaddy scripting language for last elimination of architecture and information.

- To guarantee information protection at every home stage and godaddy stage prior than and after transferring of information.

\subsection{Methodology of Purposed Research}

To ensure the successful migration of the data along with the architecture, there is a need to develop a platform which can interact with both the platforms simultaneously. It will be ensured the successful implementation of the TCP-IP protocol of one service into the local server so that its configuration can be merged into protocolling service would be used and will check that whether that service is working properly with the SAAS service of the cloud platform or not. Then there would be fetching the architecture first from the home server with the use of XML soap service to ensure the creation of the basic building block of the architecture to the another server. The XML fetched architecture would be run to the GoDaddy platform the second server [2]. To create an intermediate service for the communication, SOAP to check whether implemented service (intermediate) is able to communicate with GoDaddy or not [12]." 


\section{- FLOW CHART}

The proposed work will go as according to follows diagram:

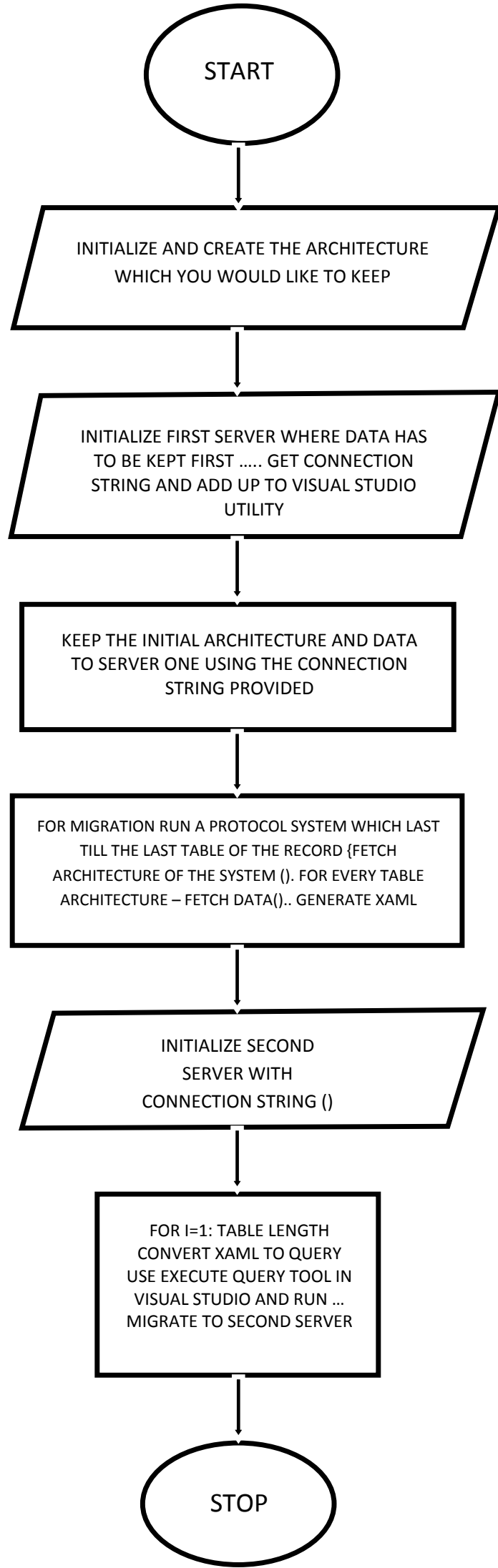

Figure 2 Proposed Flow Diagram of Data Migration Process

\section{ALGORITHM:}

A framework is designed in which all info would be shifted along with architecture of simple database method to GoDaddy method.

All work will be completed in stages as mentioned in following section.

- For cloud service create XML architecture utilization of CART ALGORITHM.

- Contain XML documents into local framework.

- Get information from XML documents to create GO Daddy script for this.

- Create Go Daddy draft for Go Daddy cloud.

Begin Go Daddy draft to Go Daddy utilizing of SVM.

\section{RESULT}

\section{Migration Process Wizard}

Migration process Wizard shows the migration process of database from one server to another server in the process the migration is done with its architecture. In this process the database is migrated without any failure.

Figure 3 Shows the Wizard that is used to migrate the schema from the primary server to the secondary server. User can migrate the whole database in a single time and if the user wants to migrate the selected schema then that is also possible. All the schema can be migrate one by one. That is user dependent what he/she want to do after the successful migration user can delete the old database from the primary server complete database or the schema that he migrate from the primary server to secondary server. After the successful migration the message is show whether the data is migrate or not. If the table or the database is migrated then the Message shows that the schema is already migrated. 


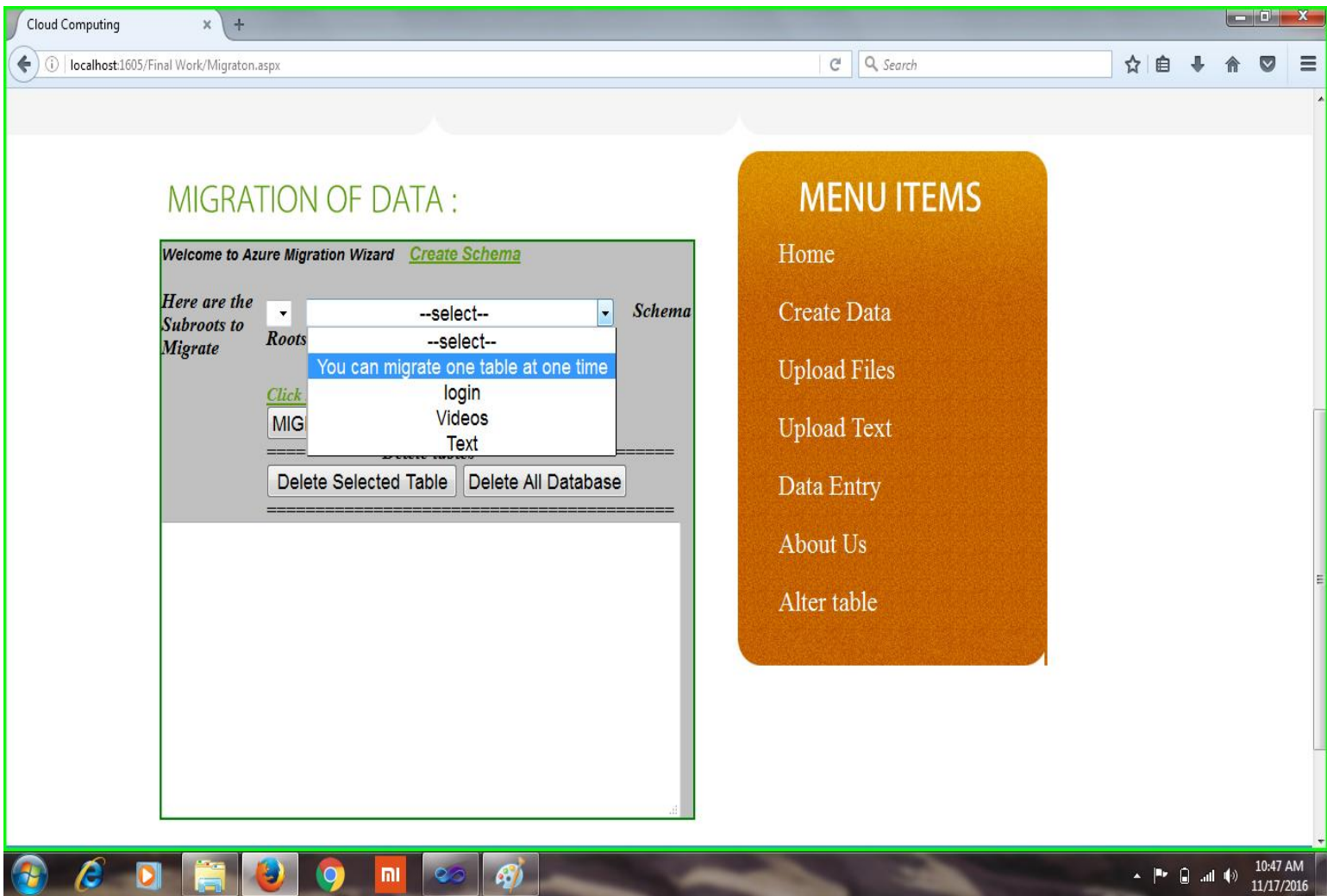

Figure 3 Migration Process Wizard

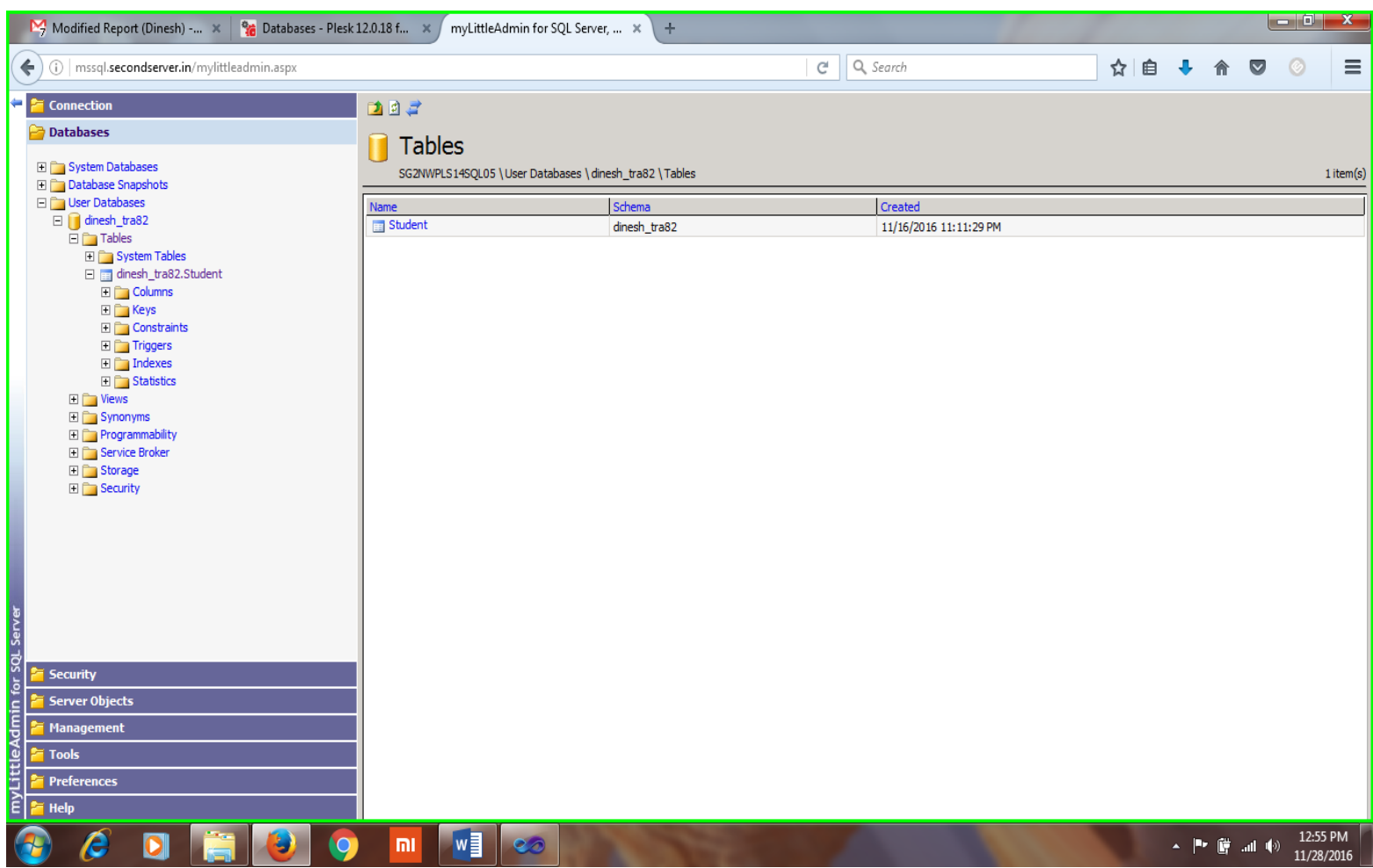

Figure 4 Secondary Server after Migration

Figure 4 shows the Secondary Server after migration in this server database student table is that table that was shown in the primary database this table is migrated from the primary server to the secondary server. This secondary server contain only student table with the architecture same as the primary database. This table shows the successful data migration from the primary server to secondary server.

\section{CONCLUSION}

Using this tool any User can migrate there data from one server to another server with its architecture. There is no need to create the architecture on new server. Secure transmission of information effectively and immediately because of buffering. The XML organize," since it can bolster any stage any sort of database can be transmitted utilizing it which will 
spare time of change of information before exchanging to customer. The above talked about work can be utilized for secure transmission of information proficiently and immediately because of buffering. The XML organize, since it can bolster any stage any sort of database can be transmitted utilizing it which will spare time of transformation of information before exchanging to customer. The information which we are moving from source to goal i.e., between heterogeneous stages is touchy (conveying data about database table and its comparing blueprint), so it must be secured. As numerous clients can get to the server at the same time, synchronization must be finished.

\section{FUTURE SCOPE}

This process is done only two server in future the process can be checked on more than two server. During the migration process if the connection is break due to internet the whole database can be migrated again.

\section{REFERENCES}

[1]. Dinesh Kumar, Dr. Praveen Dhayni \& Dr. Anuj Kumar. A Paper on the topic "Cross Breed Data Migration using ACO Algorithm" in International Journal of Computer Applications (IJCA)Volume 116 - Number 1, April 2015 ISBN: 973-93-80886-12-1(@ 2015 by IJCA Journal)

[2]. Dinesh Kumar, Dr. Praveen Dhayni \& Dr. Anuj Kumar.A Paper on the topic "Migration of Data from One Cloud Server to Another Cloud Server" in the IJAREAS International Journal of Advanced Research in Engineering and Applied Sciences, Volume 3, No. 3, March 2014, ISSN: 2278 6252,

[3]. Dinesh Kumar, Dr. Praveen Dhayni \& Dr. Anuj Kumar. A Paper on the topic "Review: A Data Migration from One Cloud to Another" in International Conference on Emerging Technologies (ICET-2014) Organized by N.C COLLEGE OF ENGINEERING, ISRANA, PANIPAT (INDIA) (April 24 - 26, 2014).

[4]. Lloyd W, Pallickara S, Olaf D, Jim L, Mazdak A, Ken R (2011) Migration of multi-tier applications to infrastructure-as-a-service clouds : an investigation $\mathrm{u}$ sing kernel-based virtual machines, Proceedings of the 2011 IEEE /ACM 12th International Conference on Grid Computing, p.137 -144, September 21-23, 2011. doi:10.1109/Grid.2011.26

[5]. Math er T, Kumaraswamy S, Latif S (2009) Cloud security and privacy. O' Reilly Media, Inc., Sebastopol, CA Menzel M, Ranjan R (2012) Cloud Genius: decision support for web server cloud migration, In: Proceedings of WWW 2012, April 16-20, 2012. Lyon, France

[6]. Mohagheghi P, Saether T (2011) Software Engineering Challenges for Migration to the Service Cloud Paradigm Ongoing Work in the REMICS Project. IEEE World Congress on Services

[7]. Pahl C, Xiong H, Walshe R (2013) "A comparison of On-Premise to Cloud Migration Approaches- A Tale of Four Cloud Migration Processes". Proc. European Conf. Service-Oriented and Cloud Computing
[8]. Parastoo M, Jørgen BA, Sadovykh A, Barbier F, Benguria G (2010) Reuse and Migration of Legacy Systems to Interoperable Cloud Services-The REMICS Project. In Proceedings of 4th Workshop on Modeling, Design, and Analysis for the Service Cloud (MDA4ServiceCloud2010), Paris, France.

[9]. Rashmi R, Shabana M, Sahoo G (2013) "Efficient migration of application to clouds: analysis and comparison ”. GSTF J Comput (3) (ISSN: 2010-2283) doi:10.5176/2251-3043_3.3.272.

[10].Razavian M, Lago P (2011) “ A Survey of SOA Migration in Industry”. Proc. Ninth Int'l Conf. ServiceOriented Computing, pp.618-626

[11].Rosado DG, Gómez R, Mellado D, Fernández Medina E (2012) Security analysis in the migration to cloud environments. Future Internet 4(2):469 -487, 10

[12].Shepperd M, Schofield C (1997) Estimating software project effort using analogies. IEEE Trans Software Eng $23: 736-743,33,51,53$

[13].Tak BC, Bhuvan U, Anand S (2011) “ To Move or Not to Move: The Economics of Cloud Computing" , Third USENIX Workshop on Hot Topics in Cloud Computing (HOTCLOUD 2011). Portland, Oregon

[14].Tran V, Keung J, Liu A, Fekete A (2011) “ Application migration to cloud: a taxonomy of critical factors ". Proc. Second Int' 1 Workshop Software Eng. For Cloud Computing

[15].Tran V, Keung JW, Liu A, Fekete A (2011b) “ Size estimation of cloud migration projects with cloud migration point ( CM P)" International Symposium on Empirical Software Engineering and Measurement ( ES EM 2011). Banff, Alberta, Canada

[16].Vu QH, Asal R (2012) “ Legacy application migration to the cloud: practicability and methodology", SERVICES, 2012 IEEE Eighth World Congress on Services, pp. 270 - 277. doi:10.1109/SERVICES.2012.47

[17]. Ward C, Aravamudan N, Bhattacharya K, Cheng K, Filepp R, Kearney R, Peterson B, Shwartz L, Young C (2010) Workload migration in t o c louds - challenges, experiences, opportunities. IE EE CLOUD 2010, Florida, U SA, pp 164- 171

[18]. Yam C Y, Baldwin A, Shiu S , Ioannid is C (2011) “ Migration to cloud as real option: investment decision under uncertainty," trust , security and privacy in computing and communications (TrustCom), 2011 IEEE $10^{\text {th }}$ International Conference on, vol., no ., pp .940,949, 16 - 18 N o v. 2011 .d oi:1 0 . 11 09/ Trust Com.2 011 .130

[19].Yu D, Jian W, Bo H, Jianxiao L, Xiuwei Z, Keqing H, Liang-Jie Z (2011) “ A practical architecture of cloud identification of legacy applications, " services (SERVICES), 2011 IEEE World Congress on, vol., no., pp.17,24, 4 - 9 July 2011. 